\title{
THE TWENTY-EIGHTH REGULAR MEETING OF THE SAN FRANCISCO SECTION.
}

THE twenty-eighth regular meeting of the San Francisco Section of the Society was held at the University of California on Saturday, November 25, 1916. The total attendance was nineteen, including the following members of the Society:

Professor R. E. Allardice, Dr. B. A. Bernstein, Professor H. F. Blichfeldt, Dr. Thomas Buck, Professor G. C. Edwards, Professor L. M. Hoskins, Dr. Frank Irwin, Professor C. J. Keyser, Professor D. N. Lehmer, Professor W. A. Manning, Professor C. A. Noble, Professor E. W. Ponzer, Professor T. M. Putnam.

Professor D. N. Lehmer was elected chairman of the Section and presided at the meeting. Professor W. A. Manning was elected secretary and Professors Blichfeldt, McDonald, and Manning members of the programme committee of the Section for the ensuing year.

The following papers were presented at this meeting:

(1) Professor W. A. Manning: "The degree and class of multiply transitive groups."

(2) Professor W. A. Manning: "The primitive groups of class fifteen."

(3) Miss Edna Johnston: "Graphical determination of the nature of the roots of cubic and quartic equations."

(4) Miss Maurine Laber: "Concerning the combinations of 36 elements 6 at a time."

(5) Professor A. F. Carpenter: "Some fundamental relations in the projective differential geometry of ruled surfaces."

(6) Professor C. J. KeYser: "A note concerning groups of dyadic relations."

(7) Professor L. M. Hoskins: "The theory of strain of an elastic solid from a condition of great initial stress; with application to two cases of strain of a gravitating sphere."

(8) Mr. J. S. TAYLOR: "Complete existential theory of Bernstein's set of four postulates for Boolean algebras."

(9) Professor H. F. Blichfeldt: "Linear groups which contain transformations having only two distinct characteristic roots."

The papers of Miss Johnston and Miss Laber were communi- 
cated to the Society by Professor Griffin. Mr. Taylor was introduced by Dr. Bernstein. In the absence of the authors the papers of Miss Johnston, Miss Laber, and Professor Carpenter were read by title.

Abstracts of the papers follow below. The abstracts are numbered to correspond to the titles in the list above.

1. Professor Manning presented the following theorem: Let $n$ be the degree and $\mu$ the class of a multiply transitive substitution group $G$ that contains a substitution of order 2 and degree $\mu$; then if the transitivity of $G$ is

$\begin{array}{ll}\text { double, } & \mu \gg \frac{1}{2} n-\frac{1}{2} \sqrt{n}-1 ; \\ \text { triple, } & \mu \geqq \frac{1}{2} n ; \\ \text { 4-ple, } & \mu \geqq \frac{1}{2}(n+1) ; \\ \text { 5-ple, } & \mu \geqq \frac{3}{5} n ; \\ \text { 6-ple, } & \mu \gtreqless \frac{3}{4} n ; \\ \text { 7-ple, } & \mu \geqq \frac{1}{1} n ; \\ \text { more than }\left(p_{1}+p_{2}+\cdots+p_{r}\right) \text {-ple, where } p_{1}, p_{2}, \cdots,\end{array}$ $p_{r}$ are distinct odd primes $(r>1)$,

$$
\mu>\frac{\left(p_{1}-1\right)\left(p_{2}-1\right) \cdots\left(p_{r}-1\right)-1}{\left(p_{1}-1\right)\left(p_{2}-1\right) \cdots\left(p_{r}-1\right)} n .
$$

Bochert, in the proof of his theorem, makes the commutator the corner stone of the whole structure. Here the commutator is abandoned and a close study of diedral rotation groups takes its place. The results of that study may be thus summarized: If the order of a group of class $\mu$ generated by two substitutions $s$ and $t$ of order 2 and degree $\mu$ is divisible by each of the odd primes $p_{1}, p_{2}, \cdots, p_{r}$, its degree $n$ does not exceed $\mu+\mu /\left(p_{1}-1\right)$ $\left(p_{2}-1\right) \cdots\left(p_{r}-1\right)$; if the order $N$ of $s t$ is odd or is divisible by $4, n$ does not exceed $\mu+2 \mu / N$; if $N(>2)$ is twice an odd number, $n$ is not greater than $\mu+2 \mu / N+8 \mu / N^{2}$.

2. It has been known for some time that the only primitive substitution groups of class $3 p, p$ an odd prime, that contain a substitution of degree $3 p$ and order $p$ are three in number and belong to one class only, class 15 . In addition to these groups Professor Manning finds that there are just four other primitive groups of class 15. In fact, if $k$ is any positive whole number greater than three, there are three simply transitive primitive groups of degree $k^{2}$ and class $3 k$, and one simply 
transitive primitive group of degree $\frac{1}{2}(k+1)(k+2)$ and class $3 k$. The last is simply isomorphic to the alternating group of degree $k$. The others are simply isomorphic to the three imprimitive groups of degree $2 k$ whose intransitive head is the direct product of two alternating groups of degree $k$ in two different sets of letters, or is the dimidiate of the direct product of two symmetric groups of degree $k$ on distinct letters. An auxiliary theorem suggested by this problem is this: If the degree of a transitive constituent of the subgroup leaving one letter fixed in a simply transitive primitive group exceeds by two (or more) units the degree of any other transitive constituent of that subgroup, then the constituent of highest degree is a simply transitive group.

3. In this note Miss Johnston points out a simple way to determine the nature of the roots of a quartic, in the various possible cases, by considering the points in the complex plane which represent the roots of the reducing cubic and their respective square roots. Similarly for a cubic and its reducing quadratic (sextic). Certain questions relating to discriminants are also discussed.

4. In his Mathematical Essays and Recreations, Ball describes methods of finding $n(n+1)$ combinations of $n^{2}$ elements taken $n$ at a time without repetition of any pair, in the cases $n=4,5,7,8$. In the present note Miss Laber proves this to be impossible in the case $n=6$, reported by Ball as having "baffled all attempts to find a solution." The proof is based upon a very convenient symmetrical system of notation, and a method of enumeration by which combinations involving common pairs can be rapidly eliminated. The number of admissible combinations resulting from various types of substitutions is noted.

5. In his projective theory of ruled surfaces, Wilczynski makes frequent use of a moving tetrahedron of reference, the homogeneous coordinates of whose vertices, when referred to any fixed system of homogeneous coordinates, are solutions of the system of differential equations

$$
\begin{aligned}
& y^{\prime \prime}+p_{11} y^{\prime}+p_{12} z^{\prime}+q_{11} y+q_{12} z=0, \\
& z^{\prime \prime}+p_{21} y^{\prime}+p_{22} z^{\prime}+q_{21} y+q_{22} z=0 .
\end{aligned}
$$


If a point, line or plane is to be fixed in space, its coordinates when referred to this moving tetrahedron must satisfy certain conditions expressible as differential equations, and conversely. Professor Carpenter's paper (a) determines necessary and sufficient conditions for fixed points, lines and planes and $(b)$ indicates by a number of theorems the usefulness of these conditions in enriching the theory of ruled surfaces. A portion of this paper was communicated to the Chicago Section of the Society at the meeting of April, 1915.

6. The abstract relations studied in symbolic logic fall into various cardinal classes. The relations of a class are combinable in accordance with certain fundamental rules or modes, as logical addition, logical multiplication, relative multiplication. Professor Keyser's paper points out the desirability of examining the more important systems thus arising with a view to ascertaining which of them are groups. The paper presents some results found on the threshold of such an examination. It is found, for example, that under any of the mentioned rules of combination the ensemble of relations that constitute a type (as this term is understood in the Principia Mathematica of Whitehead and Russell) possesses the group property and two but only two of the other properties required in the four-property definition of a group. On the other hand, the ensemble of one-one relations that cover and are restricted to an arbitrarily chosen field of relations is a group if the rule of combination be relative multiplication, though the same ensemble does not possess even the group property if the rule of combination be logical addition or logical multiplication.

7. The earlier papers upon the strain of a gravitating elastic sphere were based upon a defective physical theory, in that the stress-strain relations were assumed to hold for a fixed element of space rather than for a definite element of matter. This defect was pointed out by Professor Hoskins in 1910 (Transactions, volume 11, page 504) and by A. E. H. Love in 1911 (Some Problems of Geodynamics, page 89). An amended theory was stated by Love and applied to certain problems including that of the tides in a gravitating sphere of uniform density. The present paper is based upon a theory in essential agreement with that of Love but formulated in a somewhat 
more general manner. Professor Hoskins gives two applications: (1) to the problem of the strain of a gravitating compressible sphere of uniform density; and (2) to that of a gravitating incompressible sphere composed of a shell and nucleus of different densities. Both solutions follow the lines of the analysis employed in two former papers (Transactions, volume 11, page 203 and page 494). The same method can be extended to more complex cases such as that of a sphere composed of several shells and a nucleus, all of different densities and rigidities, such extensions being merely a matter of algebraic labor.

8. The complete existential theory of Dr. Bernstein's set of four postulates for Boolean algebras is shown by Mr. Taylor to consist of six propositions of non-existence and ten propositions of existence. The non-existences are expressed by the proposition $\Sigma^{-1} \supset \Sigma^{34}$. The propositions of existence are proved by the exhibition of ten systems $\Sigma$ : two examples for $K$ singular, six examples for $K$ dual, and two examples for $K$ triple. The postulates are shown to be completely independent in the sense defined by Professor E. H. Moore if the minimum number of elements of the class $K$ be postulated as three.

9. Professor Blichfeldt proves that:

(a) No primitive linear group can contain a transformation of order $k>5$ whose "multipliers" (characteristic roots) have only two values. For instance, no primitive group in three variables can contain the transformation $x_{1}=x_{1}{ }^{\prime}, x_{2}=\epsilon x_{2}{ }^{\prime}$, $x_{3}=\epsilon x_{3}{ }^{\prime}$, whose multipliers $1, \epsilon, \epsilon(\epsilon \neq 1)$ are $k$ th roots of unity; $k>5$.

(b) If a primitive group $G$, say in $n$ variables, contains a transformation $S$ of order $k$, where $6>k>3$, having just two distinct multipliers, then these multipliers are each repeated $\frac{1}{2} n$ times. Moreover, $G$ contains an invariant subgroup $K$. This is of monomial type and order $2^{p}$ when $k=4$. If $k=5$, then $K$ is the direct product of say $q$ groups of order $60 \varphi$ and icosahedral type, and $n$ must be divisible by $2^{q}$.

W. A. Manning, Secretary of the Section. 